\title{
Development and preclinical testing of a novel biodegradable hydrogel vaginal packing technology for gynecologic high-dose-rate brachytherapy
}

\author{
Matthew Sean Peach, MD, PhD', Joanna Moore, MPH', Wallis Giles, BS², Justin Trainor², Tim Long, PhD³, \\ Nicholas Moon, PhD',3, Joseph E. Hylton, Timothy N. Showalter, MD, MPH', Bruce Libby, PhDl \\ 'Department of Radiation Oncology, University of Virginia School of Medicine, Charlottesville, VA, ${ }^{2}$ Department of Biomedical Engineering, \\ University of Virginia School of Medicine, Charlottesville, VA, ${ }^{3}$ Department of Chemistry, Macromolecules Innovation Institute, Virginia Tech, \\ Blacksburg, VA, ${ }^{4}$ Department of Radiology, University of Virginia School of Medicine, Charlottesville, VA, USA
}

\begin{abstract}
Purpose: We evaluated the performance of a novel hydrogel-based strategy developed for clinical use as vaginal packing using phantoms and cadavers, and to compare the hydrogel to gauze and balloon packing.

Material and methods: The biocompatible hydrogel is based on a thiol-Michael addition reaction, with delivery of reagents into the vaginal cavity using a custom-made system. Soft-cured cadavers were used for soft tissue-like mechanical properties. Two cadavers with intact uteri had magnetic resonance imaging (MRI) compatible with tandem and ovoids. For one cadaver, the temperature of the vaginal canal was measured before hydrogel application, during polymerization, and after hydrogel removal. The hydrogel packing and applicator was kept in a second cadaver, which was imaged using computed tomography (CT) and MRI. The hydrogel packing and imaging was repeated for an open multichannel MRI compatible, titanium-based vaginal cylinder placed in a post-hysterectomy cadaver.

Results: The gel reaction occurred within 90 seconds, indicating polymerization at clinical quantities with a $5^{\circ} \mathrm{C}$ increase in vaginal temperature. CT and MRI imaging identified the hydrogel readily and showed a conformance to anatomy with few air pockets. The entire hydrogel packing was readily retrieved upon completion of imaging.

Conclusions: The novel strategy for polyethylene glycol (PEG)-based hydrogel intra-vaginal packing was able to rapidly polymerize in human cadavers with minimal heat production. Delivery was efficient and able to fill the contours of the vaginal cavity and displace tissue away from the applicator axis. The hydrogel has favorable imaging characteristics on CT and MRI, and shows a potential for clinical use, warranting additional studies for the use in humans.

J Contemp Brachytherapy 2018; 10, 4: 306-314 DOI: https://doi.org/10.5114/jcb.2018.77952
\end{abstract}

Key words: brachytherapy, cervical cancer, HDR, hydrogel, intrauterine tandem, vaginal packing.

\section{Purpose}

Cervical cancer brachytherapy has seen significant advances in the past decade through the incorporation of magnetic resonance imaging (MRI) into a 3D planning workflow. MRI imaging has been applied as the primary intra-procedural imaging modality [1] as well as via external MRI registration of computed tomography (CT) guided treatments [2]. The result of this improved imaging guidance has resulted in superior dosimetry [1], decreased toxicity [3], and improved outcomes [4,5]. During this period, however, there has been comparatively little evolution to applicators and accessories. One area of the treatment workflow that could be improved and have a resulting impact both on procedural efficiency and dose/toxicity to the organs at risk (OAR) is vaginal packing. Traditionally, vaginal packing utilized gauze; however, the technique is cumbersome to physicians and time consuming, which is inconvenient for patients. Further, the application and removal of gauze is frequently painful to the patient. Recently, balloon packing has improved workflow efficiency and patient comfort, allowing rapid application that has also demonstrated improved dosimetry to OAR $[6,7,8]$. However, the commercially available balloon packing options adds significant cost to the procedure and can be difficult to place in the patient's vagina. Our group therefore has focused on developing a new approach for vaginal packing that will combine the low-cost of gauze packing and the ease of utilization, efficiency, and improved vaginal wall spacing of balloon systems. 
To meet our goal, we identified a polyethylene glycol (PEG)-based hydrogel to use as a candidate for vaginal packing strategy. Hydrogels are polymers that are water insoluble due to their cross-linked network but have hydrophilic residues that promote significant water swelling. Hydrogel materials can be generated with a wide range of physical and chemical properties, based on starting subunits and reaction conditions. For biomedical applications, hydrogels are a promising material as they can attain tissue like physical proprieties, can be formed under mild conditions, and can use mild solvents including water, making them biocompatible and if designed appropriately, biodegradable.

As previously described [9], we used commercially available oligomers to develop a two-part solution that when activated via mild base $\left(\mathrm{NaHCO}_{3}\right)$, created a biodegradable hydrogel based on a thiol-Michael addition reaction. The concentration of the base used in the precursor solution allowed various reaction times and mechanical properties. The solutions and polymer precursor molecular weight were tuned such that a hydrogel was able to gel rapidly (less than 90 seconds), while attaining enough modulus (inelasticity) to displace tissue. Attaining a modulus greater than $10 \mathrm{kPa}$ (the force reached during vasalva) allows the hydrogel to have enough structural integrity to hold shape under tissue forces and potentially distend tissue during polymerization and swelling. Further, this hydrogel was shown to be biocompatible in in-vitro culture. The material has a tissue like density, which makes it easily distinguished from brachytherapy applicators on CT. However, due to the high-water content, it is easily demarcated on T2 MRI [9].

In the present study, we evaluated the performance of the hydrogel packing solution at clinically relevant reagent volumes using phantom models and cadavers. We compared the imaging appearance of hydrogel packing material to gauze and balloon packing on CT and MRI with brachytherapy applicators in place. Also, we estimated the potential application of the hydrogel material for vaginal brachytherapy using a fixed geometry multichannel applicator, with comparison to a single chan- nel vaginal cylinder applicator. The ultimate goal of this method is to provide a self-expanding hydrogel method of vaginal packing for intracavitary brachytherapy that provides reliable displacement of bladder and rectum, serving as an alternative to gauze or balloon packing during brachytherapy.

\section{Material and methods}

\section{Polymer hydrogel synthesis and delivery}

The hydrogel development has been previously described [9]. Briefly, the hydrogel synthesis involves two separate solutions that are initially prepared in separate containers (solution A and solution B). Solution A consisted of THIOCURE-ETTMP (ethoxilated-trimethylolpropan tri (3-mercaptopropionate) ) 1,300 in a $\mathrm{NaHCO}_{3}$ base, and solution $\mathrm{B}$ consisted of PEGDA (poly (ethylene glycol) diacrylate) 700 in a $\mathrm{NaHCO}_{3}$ base, delivered at a $1: 1$ ratio. The solutions are loaded into separate chambers of a commercially available two-part mixing applicator for the phantom study. A custom applicator was constructed for cadaveric studies to adapt to the larger reagent volumes and the need to reach the proximal vagina. This device consisted of two 150 cc syringes, one holding solution $\mathrm{A}$ and the other holding solution $\mathrm{B}$ that were inserted within a custom-fabricated syringe apparatus (Figure 1). The tip of each syringe connects via silicone tubing to a $Y$ connecter that attaches to a mixing chamber, which terminates in a $14 \mathrm{~cm}$ long static mixing nozzle. The syringe apparatus also includes a 3D printed plate that mounts to both syringe plungers, and allows equal dispersal of both solutions and $1: 1$ mixing in the mixing chamber, when pressure is applied (Figure 1). Previous work has shown that a $1: 1$ ratio does not need to be precisely maintained for the polymerization reaction to proceed.

\section{Phantoms}

Nylon cylindrical phantoms of the vaginal canal were fabricated using $3 \mathrm{D}$ printing. The cylinders were designed to provide a cavity measuring $3-4 \mathrm{~cm}$ in radius
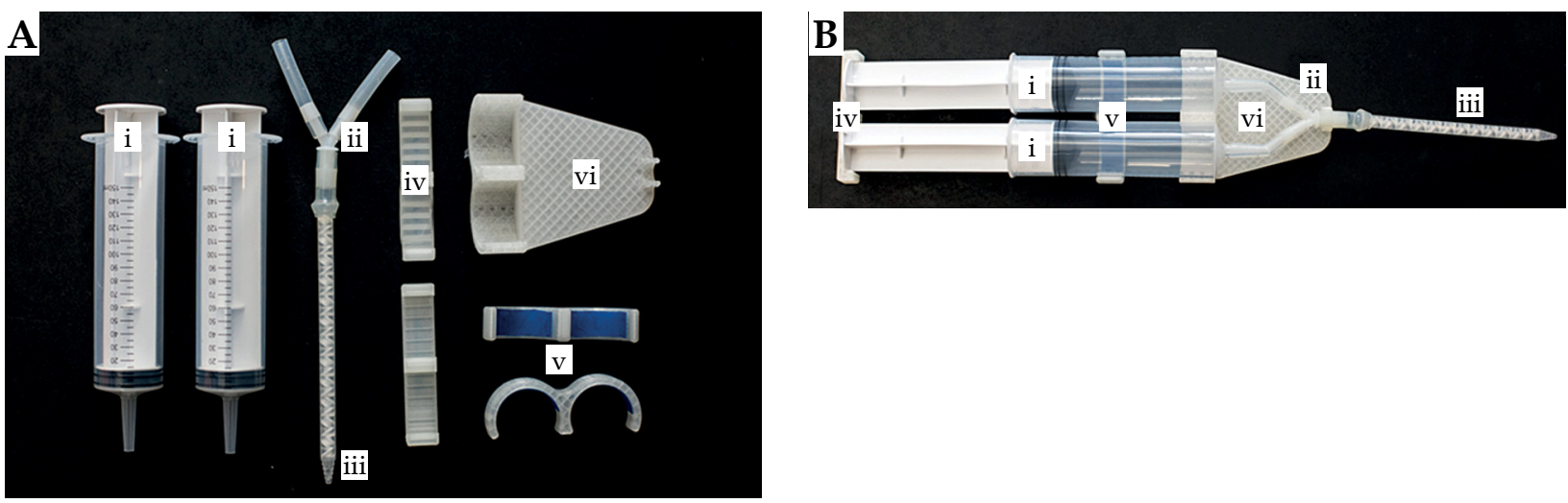

Fig. 1. Hydrogel delivery device. The device consists of (A) two $150 \mathrm{~mL}$ syringes (i), attached to silicon tubing to a Y-connector (ii) and a static mixing nozzle (iii). Syringes are held together by a 3D printed clamp to the syringe end and Y-connector (iv), a clamp to the syringe body (v), and a clamp that secures the plungers together for $1: 1$ volume mixing (iv). When fully assembled (B), this design permits delivery of solution A and solution B at 1:1 amounts using a clinically relevant quantity of reagents 

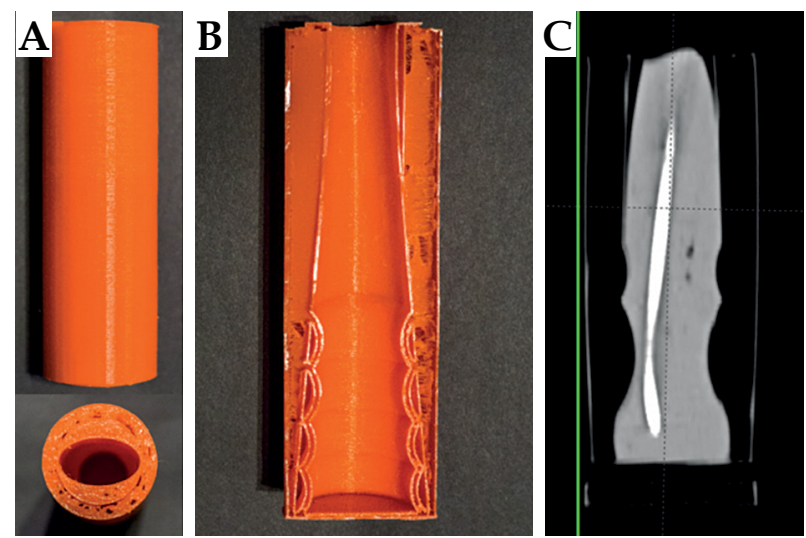

Fig. 2. A) Phantoms used to simulate vaginal anatomy for testing of hydrogel expansion. B) A cross-sectional view of a phantom displays undulations within the inner lumen of distal portion of the phantom. A coronal computed tomography image (C) demonstrates the expansion of the hydrogel to conform to the undulations within a phantom and wall distention from the polymerization as indicated by the bending of the wall relative to the green line

and $14 \mathrm{~cm}$ in length, with undulations along the inner surface to simulate normal anatomical contours (Figure 2). The structure was designed so that the walls were flexible to simulate tissue to detect distention of the cavity from expansion of the gel during the reaction. A single tandem was placed into each phantom to permit imaging of the gel next to a brachytherapy applicator. For phantom experiments, the hydrogel reaction solutions were inserted using a commercially available dual chamber epoxy applicator for an in-situ gel reaction within the phantom. CT imaging was performed for each phantom experiment.

\section{Cadavers}

In order to test packing material performance in soft tissue, we performed testing in cadavers preserved by the Thiel soft tissue embalming method $[10,11]$, a method that yields more realistic tissue mechanics. The Thiel embalming process involves the use of water, glycol, and salts as the primary embalming agents, with only very low concentrations of formaldehyde, chlorocresol, and morpholine [10]. Soft-cured cadavers provide musculoskeletal tissue flexibility with long lasting preservation of tissue quality at room temperatures, making them useful for surgical skills training and clinical simulation [11]. Traditional embalming methods, which primarily use organic solvents such as concentrated formaldehyde, have a greater degree of tissue fixing that causes tissue rigidity and unnatural mechanical responses to devises. Therefore, the soft-cured cadavers used in this study are preferable for simulation of vaginal packing material performance.

A total of two soft-cured cadavers were used in this study. A soft-cured cadaver with an intact cervix and uterus was used to evaluate vaginal packing performance with an MRI compatible tandem and ovoid brachytherapy applicator (Varian Medical Systems, Inc; Palo Alto, CA).
In a dedicated gross anatomy and surgical skills training facility, the cadaver was positioned in dorsal lithotomy and a speculum was inserted. The cervix was visualized, and the tandem was placed. The speculum was removed and the ovoids were sequentially inserted. The applicator was secured. The reagent and reactive solutions were preloaded into the above described syringe apparatus (Figure 1) with a total of $100 \mathrm{cc}$ of reagents used (50 cc per syringe). The applicator tip was inserted to the proximal vagina and the solution was steadily deposited. The tip was gradually withdrawn as the vaginal cavity was filled to the introitus (approximately 10 seconds). The tandem and ovoids were held in position for 90 seconds, after which the integrity of the hydrogel was assessed. This process was repeated with the same cadaver two more times, using gauze packing and using saline-filled balloon packing (Radiadyne; Houston, TX).

A second soft-cured cadaver without a uterus (status post-hysterectomy) was utilized to study the hydrogel packing with a prototype multichannel vaginal applicator. The multichannel applicator was a prototype consisting of 5 channels and made of titanium for MRI compatibility. For applicator insertion, the cadaver was placed in the dorsal lithotomy position. A speculum was inserted to ensure that there was an intact vaginal cuff, and then the applicator was inserted. The vagina was filled with the hydrogel as described above. On a separate trial, a standard single channel vaginal cylinder (Varian Medical Systems; Palo Alto, CA) was placed for comparative imaging.

\section{Imaging}

The phantom with hydrogel packing underwent CT imaging with a Siemens SOMATOM ${ }^{\circledR}$ sliding gantry CT (Siemens AG; Berlin and Munich, Germany) using $1.5 \mathrm{~mm}$ slice thickness. For the cadaver studies, scanning was performed on a Siemens Biograph 64 PET/CT using $1.5 \mathrm{~mm}$ slice thickness for CT imaging. Cadavers were then transferred to a $1.5 \mathrm{~T}$ Siemens Avanto MRI with T2 sequencing obtained with $2.0 \mathrm{~mm}$ slice thickness.

\section{Results}

\section{Hydrogel reaction characteristics}

Hydrogel formation occurred in less than 90 seconds in both the phantom and cadaver studies, and the hydrogel firmly stabilized the applicator suggesting that the hydrogel expands to serve as packing within a clinically acceptable time frame. The average measured increase in temperature from the hydrogel reaction was $5^{\circ} \mathrm{C}$, based upon thermometer measurements in the cavities before and after hydrogel formation. The gel also visually exhibited distention on the walls of the phantoms, suggesting a potential for tissue displacement as vaginal packing (Figure 2). The hydrogel product was readily extracted from the phantoms and cadaver cavities without excessive force or shearing of the product. The hydrogel product remained attached to the applicator for en bloc removal (Figure 3), and then was readily removed from the applicator after soaking in water. 


\section{Phantom imaging}

CT imaging of the phantoms indicate that the gel was able to readily adapt to the phantom shape and undulations (Figure 2). The hydrogel was able to conform to the phantom geometry with minimal air pockets. The uniform appearance and Hounsfield units (HU) also indicates that the hydrogel was thoroughly mixed prior to polymerization with resulting homogenous density. The polymerized hydrogel was easily distinguishable from the tandem placed in the hydrogel (Figure 2), with a Hounsfield number of approximately 100 . The uniform HU also indicates that the hydrogel was thoroughly mixed prior to polymerization with resulting homogenous density.

\section{Cadaver study with hydrogel vaginal packing}

The design of the customized delivery system for the cadaver studies was successful, being able to deliver the polymer reagents to the apex of the vagina with brachytherapy applicators in place for in situ reaction. Thus, the hydrogel was able to serve as a vaginal packing after placement of brachytherapy applicators. The apparatus was also capable to deliver the entire solution with-
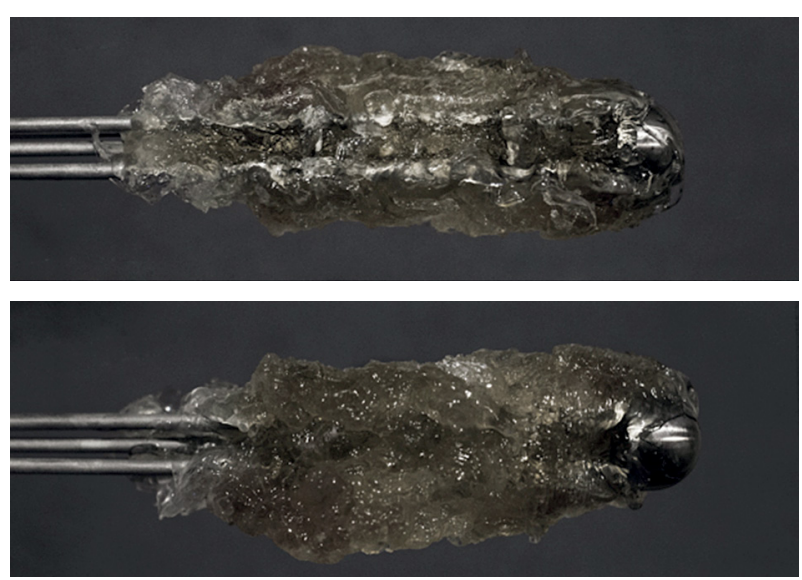

Fig. 3. Removed multichannel vaginal cylinder with hydrogel intact. The hydrogel product is removed with minimal force of extraction

out the nozzle clogging from rapid polymerization. The flow from the apparatus was controllable with the plunger clamp, obtaining a continuous and easily controllable flow with little solution spilling from the cadavers when in the dorsal lithotomy position.
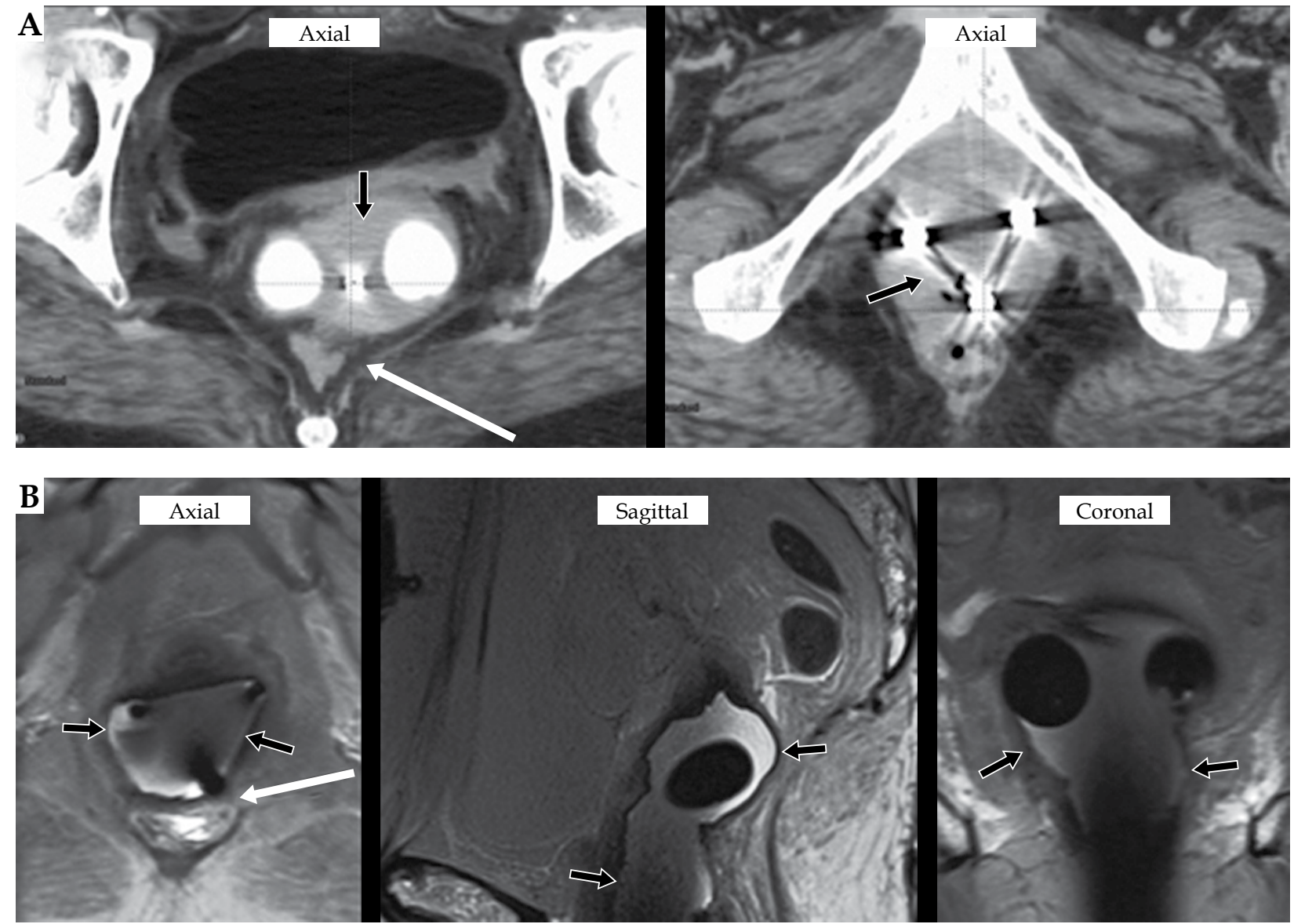

Fig. 4. Computed tomography (CT) (A) and magnetic resonance imaging (MRI) (B) of T\&O with hydrogel packing. The hydrogel results in a homogenous CT signal (A, black arrows) with the get displacing the rectum is (A, white arrow). The polymerized hydrogel results in a homogenous signal well demarcated on T2 MRI from the vaginal mucosa with tissue displacement (B, black arrows) 
CT and MRI images show that the hydrogel dispersed throughout the vagina for complete and homogenous packing with applicators in place with minimal air pockets (Figures 4 and 5). Both the multichannel cylinder and the tandem and ovoid (T\&O) applicator were readily distinguished from the gel. However, there is some visual uncertainty in defining the tissue-hydrogel interface on CT given the soft tissue density of the gel on CT (Figures $4 \mathrm{~A}$ and $4 \mathrm{~B}$ ). There was homogenous signal from the hydrogel indicating that the solutions were mixed well. MRI imaging clearly showed the hydrogel packing from the surrounding soft tissue of the vagina (Figure $4 \mathrm{~A}$ and $4 \mathrm{~B}$ ). Further, the gel was able to radially displace the vaginal tissue on average $9.16 \mathrm{~mm}$ (range, 4.63-16.04 mm) $\mathrm{cm}$ from the multichannel catheter. Tissue was well displaced radially in a uniform manor.

\section{Comparison to gauze and balloon packing for T\&O brachytherapy}

In order to provide a comparison to the hydrogel-based packing, both gauze and balloon packing were also placed in the cadavers along with the T\&O applicator. The gauze packing displaces soft tissue, and is distinguishable from adjacent soft tissue and the brachyther- apy applicator but contains air gaps within the packing material (Figure 6). There were also several areas of unequal tissue displacement. Similarly, the balloon packing contained air gaps but was more clearly seen with a homogenous T2 signal (Figure 7). The balloons achieved significant tissue displacement in certain regions of the vaginal canal but had unequal distribution, leading to areas with decrease vaginal and rectal tissue displacement. The hydrogel material demonstrated tissue displacement and imaging appearance of similar to the balloon packing with a lack of air pockets and more uniform displacement (Figures 4 and 7).

\section{Comparison to single channel vaginal cylinder brachytherapy}

Cadaver imaging studies were performed with a single channel cylinder in place for comparison to the use of a 5-channel applicator with hydrogel. Comparative images are shown in Figure 8. The standard single channel cylinder appears to better displace soft tissue than the 5-channel applicator with hydrogel. The hydrogel expanded around the 5-channel applicator for uniform distribution that would provide some tissue displacement and attenuation of radiation dose.
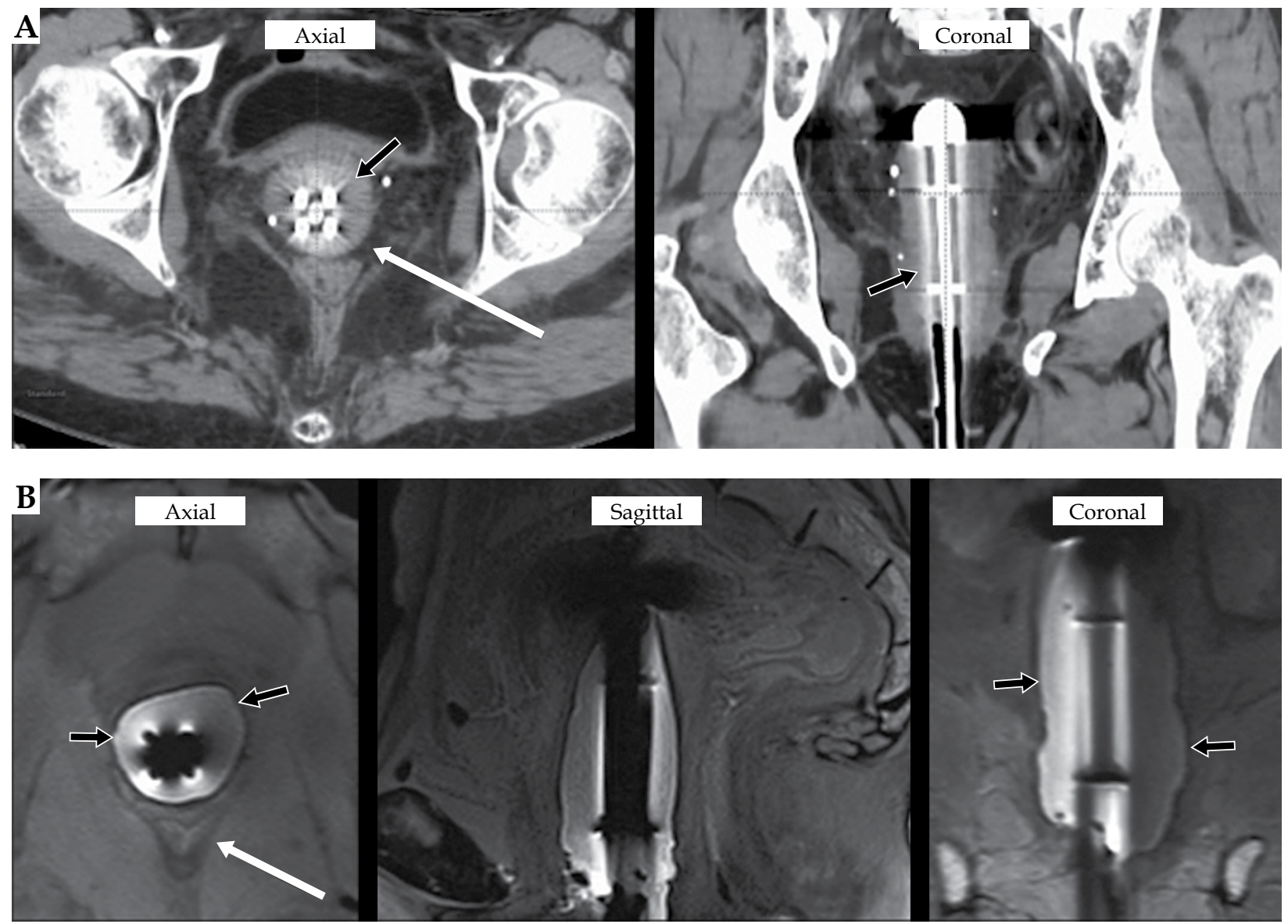

Fig. 5. Computed tomography (CT) (A) and magnetic resonance imaging (MRI) (B) of 5-channel vaginal brachytherapy applicator with the hydrogel results in a homogenous CT signal (A, black arrows) with the hydrogel displacing the rectum is (A, white arrow). The polymerized hydrogel results in a homogenous signal well distinguished on T2 MRI from the vaginal mucosa (B, black arrows) with displacement of the rectum (B, white arrows) 


\section{Discussion}

Our evaluation of the performance of a PEG-based hydrogel in phantoms and cadavers provides strong preclinical evidence to support further development of this strategy for using this hydrogel as vaginal packing for gynecological brachytherapy. Using clinically-relevant hydrogel volumes, we showed that hydrogel reaction occurs quickly and with minimal heat production, conform well to the phantom or cadaveric vaginal cavity, has favorable imaging properties on CT and MRI, and can be easily removed after using with no excessive force during extraction. The reaction time and performance of the hydrogel is appropriate for our intended goal to provide a hydrogel packing method that expands within the vaginal cavity, serving as vaginal packing, with the goal of relatively automatic expansion with no need for adjustment of the packing during placement. We did not perform a dosimetric analysis in the current study, since only one cadaver was used for each method and our images suggest similar displacement of adjacent tissue among all three packing methods. Our findings suggest the hydrogel is a promising alternative for customized vaginal packing that can be developed as a less expensive product than commercially available balloon packing options. Additional research will be required to develop this approach for clinical use, including biocompatibility testing, fabrication and testing of a delivery system to support consistent instillation into the vaginal cavity, clinical trials in humans, and regulatory clearance.

Although the observed expansion properties and temperature changes may be adequate for clinical use, it will be important to confirm the current findings in human studies. Based on the available literature on thermal pain, the temperature rise in the current study would not be expected to cause patient discomfort [12]. Since blood flow may help to disperse heat, the temperature rise associated with the gel reaction may be less than the $5^{\circ} \mathrm{C}$ change observed in cadavers. The ability of the hydrogel to displace tissue will need to be establish in-vivo, since its use as vaginal packing is dependent upon the ability to expand to move bladder and rectum from the brachytherapy applicator. If there is a need to optimize the degree of expansion during the hydrogel reaction, the kinetics of the thiol-Michael addition reaction can be tuned by adjusted reagent concentrations [9]. This strategy is intended to deliver self-expansion of the hydrogel product
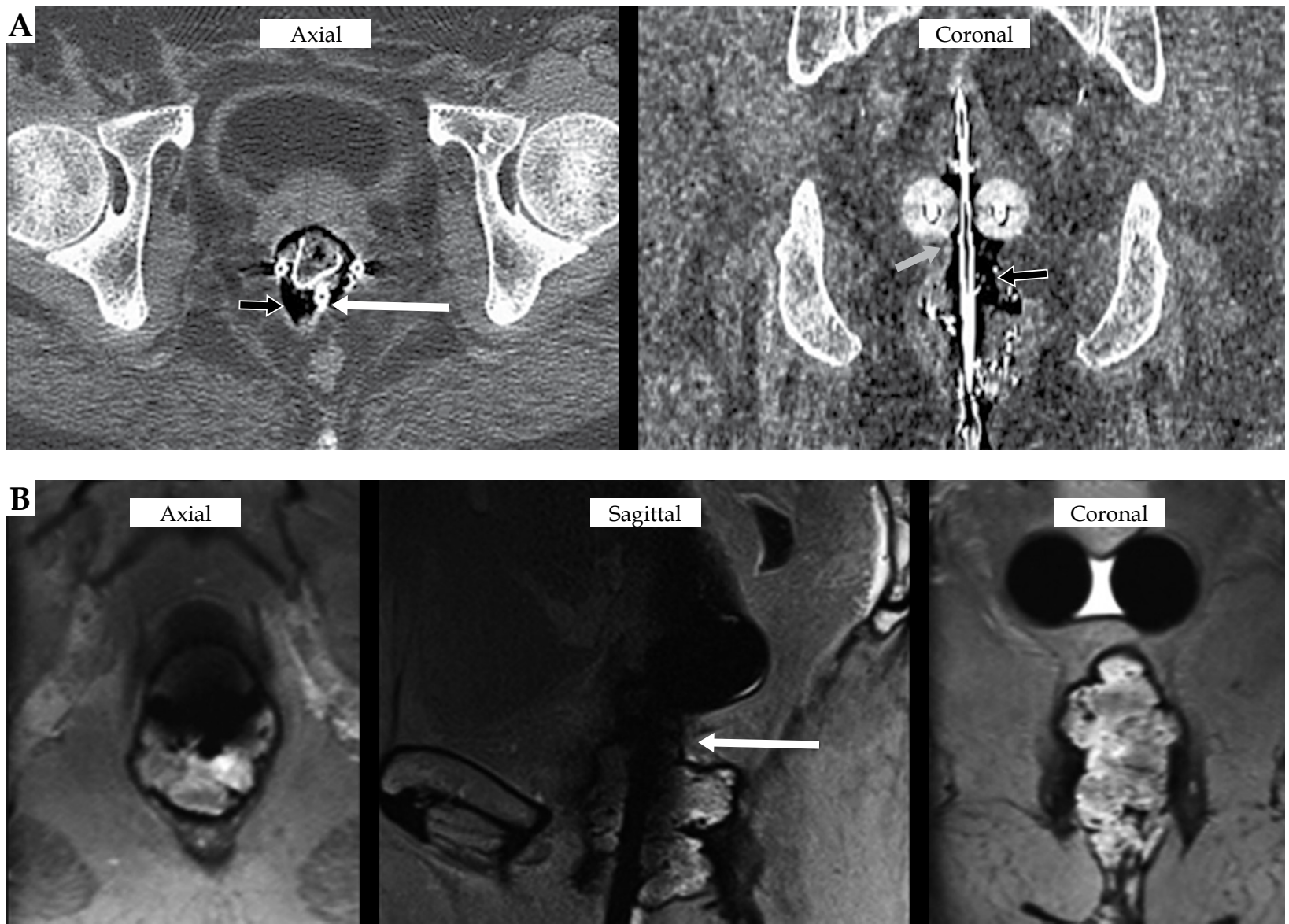

Fig. 6. Computed tomography (CT) (A) and magnetic resonance imaging (MRI) (B) of T\&O with gauze packing. Gauze packing results in significant air pockets (A, black arrows) and areas with unsuccessful displacement of the rectum (A, white arrow) and vaginal tissue (A, gray arrow). T2 MRI imaging further demonstrates area of poor tissue displacement with less than uniform packing (B, white arrow) 

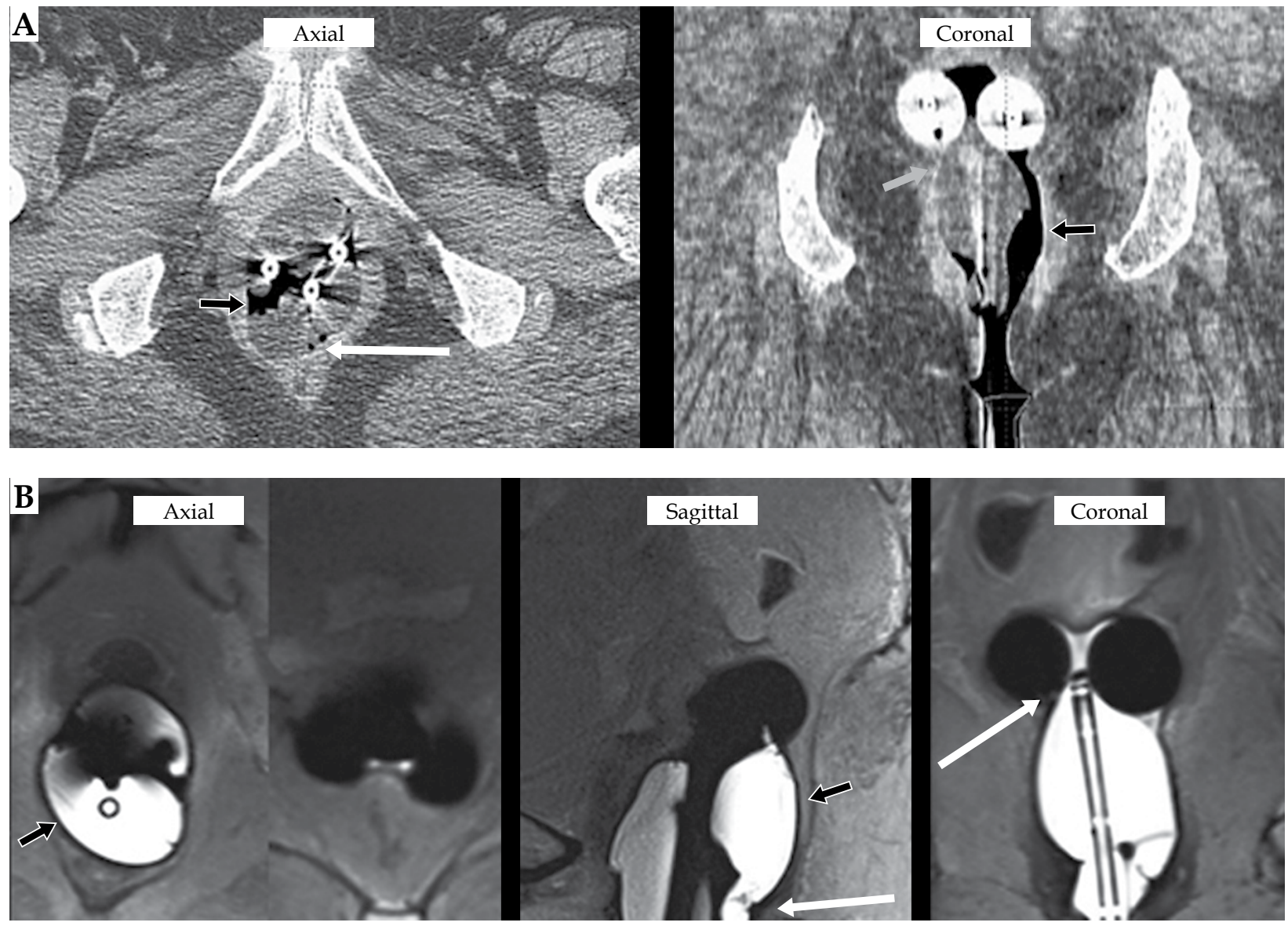

Fig. 7. Computed tomography (CT) (A) and magnetic resonance imaging (MRI) (B) of T\&O with balloon packing. Balloon packing results in concentrated regions of air pocket (A, black arrows) and areas with partial displacement of the rectum (A, white arrow) and vaginal tissue (A, gray arrow). T2 MRI imaging more clearly demonstrates unequal distribution of the balloon packing (B, black arrows) with areas of poor tissue displacement (B, white arrows)

in the vaginal cavity without need or opportunity for the clinician to revise the packing during the brachytherapy procedure. Therefore, chemical reagents that would provide a rapid hydrogel reaction were chosen. Finally, although preliminary testing suggests that this hydrogel strategy produces a biocompatible product [9], it will be essential to confirm this in additional experiments to establish its safety in humans. Although PEG-based hydrogels are use in humans, including as prostate-rectal spacer for prostate radiation therapy [13], we are not aware of the thiol-Michael addition reaction being used for medical implants.

Our comparative study focused on gauze and balloon packing for the comparisons in cadavers, as we envision developing this hydrogel method as a packing material for temporary placement during intracavitary brachytherapy. Others have previously described using a different hydrogel strategy to reduce rectal doses during cervical cancer by using a needle to inject hydrogel into the vaginal fornices and rectovaginal septum with a hydrogel material that is commercially available for prostate cancer radiation therapy $[13,14,15,16]$. Similarly, Basu et al. described needle injection of hydroxypropyl methylcellulose into the rectovaginal septum to create rectal separation for cervical cancer brachytherapy
[17]. Because our strategy focuses on the use of hydrogel for vaginal packing rather than injection of hydrogel into the rectovaginal septum, we did not compare our investigational strategy to these methods.

The imaging properties of the hydrogel on CT and MRI are favorable, with homogeneous, hyperintense signal on T2-weighted MRI due to the high-water content of the gel. The hydrogel is readily distinguishable from brachytherapy applicators and tissue. Comparison images with gauze and balloon packing suggested that the degree of packing and potential for radiation attenuation provided by the hydrogel are similar to balloon packing and better than gauze packing with significantly less air pockets. As this was a preclinical study to assess the feasibility of the use of the hydrogel in cadavers, any clinical use will have to be performed in a trial to compare the consistency of adequate packing with hydrogel versus gauze packing, as well as the potential for the in-situ expansion of hydrogel packing to better conform to a wider range of patient anatomy than balloon packing (which is a single size).

This study is intended to provide the foundation to support subsequent clinical testing and commercial development, however important limitations should be noted. First, the cadavers and phantoms do not fully reflect 

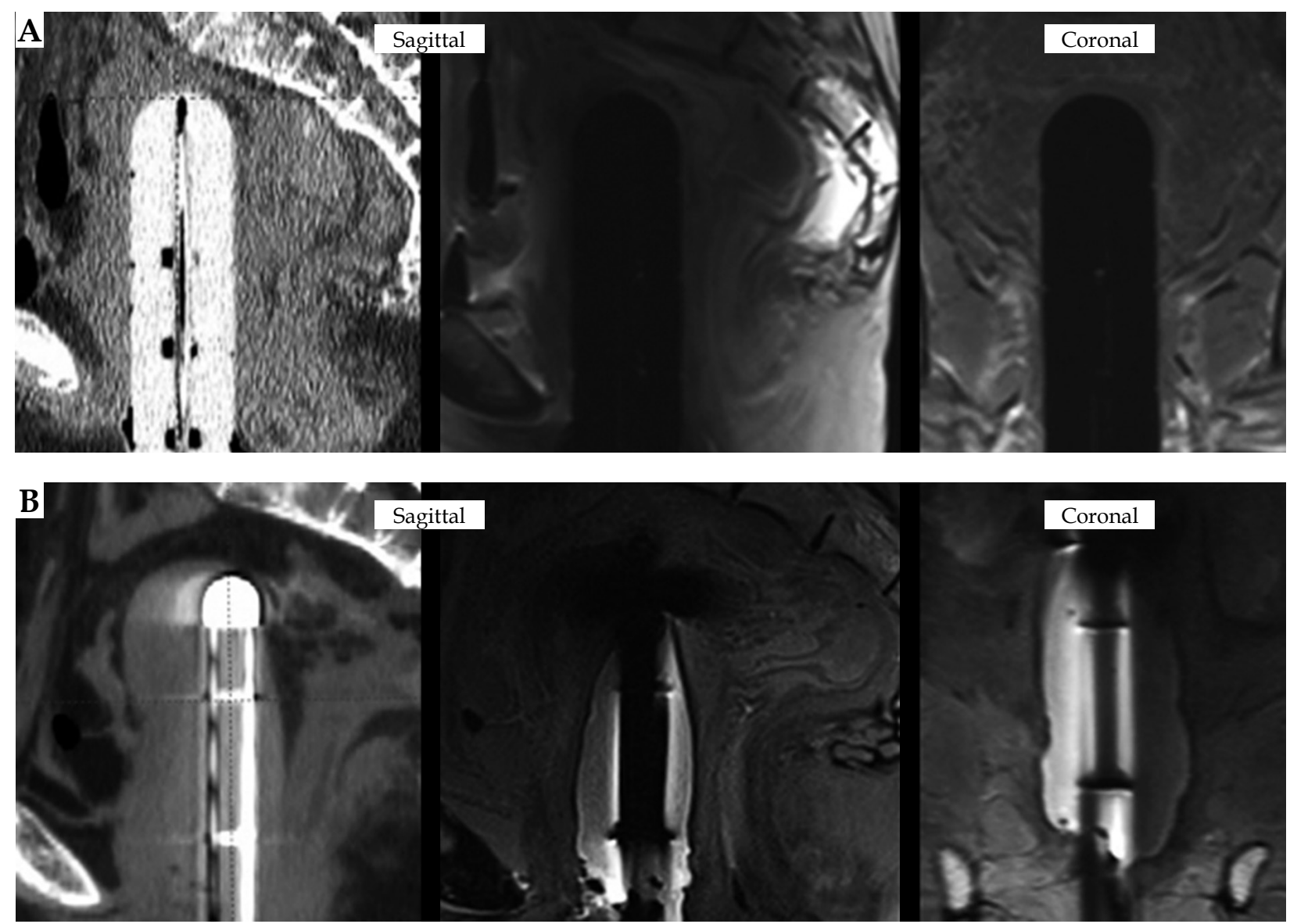

Fig. 8. Standard vaginal cylinder (A) vs. noval multichannel vaginal cylinder with hydrogel packing (B)

patient anatomy in clinical procedures in cervical cancer patients. It is possible that the amount of tissue displacement provided by the hydrogel is either not enough or too much for optimal clinical performance, which may require further adjustment of reagent concentrations during future development [9]. Although initial studies suggest that the hydrogel is biocompatible, it has not yet been fully evaluated for mucosal irritation and tissue adherence, therefore it is not yet ready for clinical use. In addition to evaluating safety in humans, future studies will be needed to determine whether hydrogel packing improves outcomes, such as patient comfort or reliability of excellent vaginal packing by physicians, compared to gauze and balloon packing.

In addition to use as vaginal packing for intracavitary cervical cancer brachytherapy with $\mathrm{T} \& \mathrm{O}$, the hydrogel was tested in the cadaver studies in conjunction with a 5-channel vaginal cuff applicator. On CT and MRI, the hydrogel appears to expand around the applicator to fill the vaginal cavity, conforming to the anatomy and providing potential for radiation attenuation. This applicator was designed for use with hydrogel for vaginal brachytherapy to provide an option for customized brachytherapy using a single applicator. Since the applicator is designed as a fixed-geometry scaffold, it would be possible to use a pre-digitized applicator and/ or library plan to facilitate rapid treatment planning for clinical use in image-guided brachytherapy. This would save time compared to disposable multi-channel cylindrical applicators, such as the Varian Capri ${ }^{\mathrm{TM}}$ applicator (Varian Medical Systems, Palo Alto, CA). Furthermore, the ability to offer customized brachytherapy through hydrogel used in combination with a single size, reusable applicator may provide the opportunity for cost-savings, compared to other alternatives such as stocking a range of different sizes of applicators or using commercially available disposable options for this purpose. We did not compare our strategy to vaginal mold techniques of vaginal brachytherapy $[18,19,20]$, but one would expect mold techniques to be more conformal, with better target volume coverage and lower doses to adjacent organs as well as more time-consuming than our proposed methodology. It should be noted that the hydrogel reaction occurs very rapidly and can be removed readily after the procedure, which may provide some workflow and patient comfort advantages compared to the vaginal mold technique. However, this is beyond the scope of our current study. Since our hydrogel-based concept for vaginal packing features a 5-channel cylinder rather than a single channel design, we expect minor dosimetric advantages over standard single-channel vaginal cylinder applicators. Future research will include developing the application method of the hydrogel packing combined with a fixed-geometry, multichannel applicator for clinical use in imageguided vaginal brachytherapy. 


\section{Conclusions}

In conclusion, the PEG-based hydrogel intra-vaginal packing was able to rapidly polymerize in phantoms and in human cadavers with minimal heat production. Delivery was efficient, able to fill the contours of the vaginal cavity and displace tissue away from the applicator axis, and removal was easy. The hydrogel has favorable imaging characteristics with a uniform, hyperintense appearance on T2-weighted MRI. Our findings suggest this hydrogel is a promising alternative to gauze or balloons for vaginal packing. Future research will be aimed at establishing biocompatibility of this hydrogel for use in humans and clinical testing of the hydrogel's performance as vaginal packing in cervical cancer patients receiving brachytherapy.

\section{Disclosure}

Research reported in this publication was supported by a grant from the Ivy Biomedical Innovation Fund. Dr. Showalter owns equity and is an employee of BrachyFoam, LLC, which is working to develop this technology for commercial use.

The authors report no conflict of interest.

\section{References}

1. Viswanathan AN, Dimopolous J, Kirisits C et al. Computed tomography versus magnetic resonance imaging-based contouring in cervical cancer brachytherapy: results of a prospective trial and preliminary guidelines for standardized contours. Int J Radiat Oncol Biol Phys 2007; 68: 491-498.

2. Trifiletti DM, Libby B, Feuerlein $S$ et al. Implementing MRIbased target delineation for cervical cancer treatment within a rapid workflow environment for image-guided brachytherapy: a practical approach for centers without in-room MRI. Brachytherapy 2015; 14: 905-909.

3. Gill BS, Kim H, Houser C et al. MRI-guided high-dose-rate intracavitary brachytherapy for treatment of cervical cancer: The University of Pittsburgh experience. Int J Radiat Oncol Biol Phys 2015; 91: 540-547.

4. Kamran SC, Manuel MM, Cho LP et al. Comparison of outcomes for MR-guided versus CT-guided high-dose-rate interstitial brachytherapy in women with locally advanced carcinoma of the cervix. Gynecol Oncol 2017; 145: 284-290.

5. Rijkmans EC, Nout RA, Rutten IH et al. Improved survival of patients with cervical cancer treated with image-guided brachytherapy compared with conventional brachytherapy. Gynecol Oncol 2014; 135: 231-238.

6. Eng TY, Patel A, Knaup C et al. Significant rectal and bladder dose reduction using intravaginal balloons during highdose-rate (HDR) intracavitary brachytherapy (ICBT) for cervical cancer. Brachytherapy 2012; 84 (3S): S445.

7. Eng TY, Patel AJ, Ha CS. Rectal and bladder dose reduction with the addition of intravaginal balloons to vaginal packing in intracavitary brachytherapy for cervical cancer. Brachytherapy 2016; 15: 312-318.

8. Rai B, Patel F, Chakraborty $S$ et al. Bladder-rectum spacer balloon versus vaginal gauze packing in high dose rate brachytherapy in cervical cancer: a randomised study (Part II). Clin Oncol (R Coll Radiol) 2015; 27: 713-719.

9. Moon NG, Pekkanen AM, Long TE et al. Thiol-Michael 'click' hydrogels as an imageable packing material for cancer therapy. Polymer 2017; 125: 66-75.
10. Eisma R, Lamb C, Soames RW. From formalin to Thiel embalming: What changes? One anatomy department's experiences. Clin Anat 2013; 26: 564-571.

11. Healy SE, Rai BP, Biyani CS et al. Thiel embalming method for cadaver preservation: a review of new training model for urologic skills training. Urology 2015; 85: 499-504.

12. Lindstedt F, Lonsdorf TB, Schalling M et al. Perception of thermal pain and then thermal grill illusion is associated with polymorphisms in the serotonin transporter gene. PLoS One 2011; 6: e17752.

13. Mariados N, Sylvester J, Shah D et al. Hydrogel Spacer Prospective Multicenter Randomized Controlled Pivotal Trial: Dosimetric and Clinical Effects of Perirectal Spacer Application in Men Undergoing Prostate Image Guided Intensity Modulated Radiation Therapy. Int J Radiat Oncol Biol Phys 2015; 92: 971-977.

14. Damato AL, Kassick M, Viswanathan AN. Rectum and bladder spacing in cervical cancer brachytherapy using a novel injectable hydrogel compound. Brachytherapy 2017; 16: 949-955.

15. Viswanathan AN, Damato AL, Nguyen PL. Novel use of a hydrogel spacer permits reirradiation in otherwise incurable recurrent gynecologic cancers. J Clin Oncol 2013; 31: e446-447.

16. Marnitz S, Budach V, Weiber F et al. Rectum separation in patients with cervical cancer for treatment planning in primary chemo-radiation. Radiat Oncol 2012; 7: 109.

17. Basu S, Manir KS, Basu A et al. Rectal separation using hydroxypropyl methylcellulose in intracavitary brachytherapy of cervical cancer: an innovative approach. J Contemp Brachytherapy 2016; 8: 399-403.

18. Magné N, Chargari C, SanFilippo N et al. Technical aspects and perspectives of the vaginal mold applicator for brachytherapy of gynecologic malignancies. Brachytherapy 2010; 9: 274-277.

19. El Khoury CE, Dumas I, Tailleur A et al. Adjuvant brachytherapy for endometrial cancer: Advantages of the vaginal mold technique. Brachytherapy 2015; 14: 51-55.

20. Nilsson S, Moutrie Z, Cheuk et al. A unique approach to high-dose-rate vaginal mold brachytherapy of gynecologic malignancies. Brachytherapy 2015; 14: 267-272. 